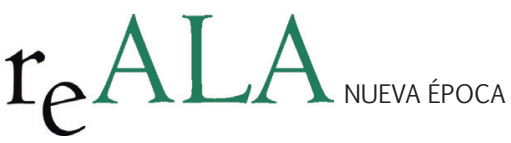

REALA, n 2, julio-diciembre 2014, ISSN: 1989-8975

DOI: http://dx.doi.org/10.24965/reala.voi2.10189

\title{
Legitimidad del proceso de participación popular: una investigación de las prácticas de planificación pública en Brasil
}

\author{
Marco Aurélio Marques Ferreira \\ Profesor Adjunto de la Universidad Federal de Viçosa (UFV-Brasil) \\ marcoaurelio@ufv.br
}

Ambrozina de Abreu Pereira Silva

Profesor Asistente de la Universidad Federal de Ouro Preto (UFOP-Brasil)

ambrozinaap@yahoo.com.br

Anderson de Oliveira Reis

Estudiante de Posgrado en Administración Pública por la Universidad Federal de Viçosa (UFV-Brasil) anderson.reis@ufv.br

\section{Resumen}

Cambios ocurridos en el escenario mundial han exigido nuevos modelos de gestión, imprimiendo una nueva relación entre Estado y Sociedad. En este sentido, existe una tendencia de la Administración Pública, antes considerada cerrada a los ciudadanos, que pasa a privilegiar la participación de estos sujetos en el proceso. En ese contexto, este trabajo tuvo como objetivo verificar cómo y cuándo se ha producido la participación popular en los procesos de elaboración, control y evaluación del Plan de Acción Gubernamental en el nivel estadual del gobierno Brasileño. El estudio utiliza como marco teórico el abordaje democrático-participativo de la Gobernanza, que busca estimular la organización de la sociedad civil y promover la restructuración de los mecanismos de decisión, en favor de una implicación más grande de la población en el control social de la administración pública y en la definición e implementación de políticas públicas.

Palabras Clave

Control, Políticas Públicas, Accountability, Transparencia

\section{Legitimacy of the Citizen Participation Process: an Investigation on Public State Planninfg Practices in Brazil OF}

\begin{abstract}
Changes that have occurred on the world have required new management models, imposing a new relationship between State and Society. In this sense, there is a tendency of Public Administration, which was considered inaccessible to citizens, to start to favor the participation of the citizens in that process. In this context, the present study aimed to verify how and when the citizen's participation occurred in the processes of elaboration, monitoring and evaluation of the Governmental Action Plan at the State level of the Brazilian government. The study had as its theoretical framework the participatory-democratic approach of the Governance, which seeks to stimulate the civil society organization and promote the restructuring of the decision-making mechanisms, in favor of higher citizen's engagement in activities of social control of the public administration and in the definition and implementation of public policies.
\end{abstract}

Keywords

Control, Public Policies, Accountability, Transparency. 


\section{INTRODUCCIÓN}

Cambios ocurridos en el escenario mundial han exigido nuevos modelos de gestión que pretenden mejorar el desempeño de la Administración Pública y del Gobierno, produciendo una nueva relación entre Estado y sociedad. Las reformas gerenciales que ocurren a partir de los años noventa sitúan como problema central la necesidad de redefinir los modelos de gestión pública a partir de nuevos modelos institucionales que permitan ampliar la capacidad de gobernar, la legitimidad política y la eficiencia en la provisión de bienes y servicios para la sociedad.

Se percibe un nuevo posicionamiento del Estado que deja de ser responsable directo del desarrollo económico y social para fortalecerse en la función de promotor y regulador. Diversos servicios públicos fueron delegados al tercer sector y a la iniciativa privada (Bresser-Pereira, 1998).

El Estado comienza a apreciar que solo no es capaz de atender las necesidades de los ciudadanos, reconociendo la necesidad de la contribución del sector privado y del tercer sector para el alcance del bienestar de la sociedad, haciendo que la administración pública pase a estar volcada con el ciudadano y enfocada a la interacción entre Estado, iniciativa privada y tercer sector.

Son entonces utilizados en el escenario de la gestión pública modelos como el democrático-participativo, el cual enfatiza la importancia de la participación de los ciudadanos en el proceso de gestión pública. La Administración Pública que antes era considerada cerrada a los ciudadanos, pasa a privilegiar la participación de estos sujetos en el proceso, así, el ciudadano, pasa de espectador y mero cliente pasivo de las prestaciones materiales de la Administración Pública a sujetos activos en el ciclo de la concepción, implantación y control de políticas públicas.

En ese contexto, el presente trabajo tuvo como objetivo verificar como ha ocurrido y cuál es el nivel de participación popular en los procesos de elaboración, control y evaluación del Plan de Acción Gubernamental en el nivel estadual del Gobierno Brasileño.

Diversos estudios han abordado la temática de participación popular, por ejemplo el trabajo de Grimmelikhuijsen (2012); Costa y Camelo (2010); Milani (2008); Fadul y Mac Allister (2007); Frey (2007); Devas y Grant (2003); Toni (2002); Teixeira, Dagnino y Almeida (2002) y Bovens (1998).

\section{REFERENCIAS TEÓRICAS}

En esta sección serán presentados los abordajes teóricos que sustentarán las discusiones propuestas en este trabajo, siendo estas: gestión pública y niveles de participación, que abordará el proceso de compromiso de la sociedad y, de forma complementaria el proceso de gobernanza en el sector público.

\section{GESTIÓN PÚBLICA Y NIVELES DE PARTICIPACIÓN}

Diversos autores, en el mundo, han abordado el creciente proceso de participación y mayor transparencia en el sector público bajo diferentes perspectivas, por ejemplo Goldfrank (2006) que estudia las experiencias de planificación presupuestaria participativa en América Latina; Kolstad y Wiig (2009) que presentan la transparencia como elemento clave en el combate de la corrupción; Prat (2005) que explora las formas erróneas de proponer la transparencia; Premchand (2001) que aborda los enfoques y la realidad en el proceso de transparencia.

Datos de diversas organizaciones, como por ejemplo OVERSEAS DEVELOPMENT INSTITUTE - ODI (2007) han destacado el aumento del proceso de transparencia en el sector público.

De acuerdo con Khagram et al. (2013) es posible destacar cuatro factores que en diferentes países impulsaron este proceso, siendo estos: a) La transición política hacia formas más democráticas de gobierno; b) Las crisis económicas y fiscales que forzaron a los gobiernos a implantar alternativas de mayor disciplina fiscal y de mayor exposición de las cuentas públicas; c) La larga exposición de casos de corrupción, lo que abrió espacios para que el ejecutivo y el legislativo introdujesen mecanismos de acceso a las cuentas públicas; y d) La presión externa y popular para la implantación de reformas domésticas (Figura 1). 


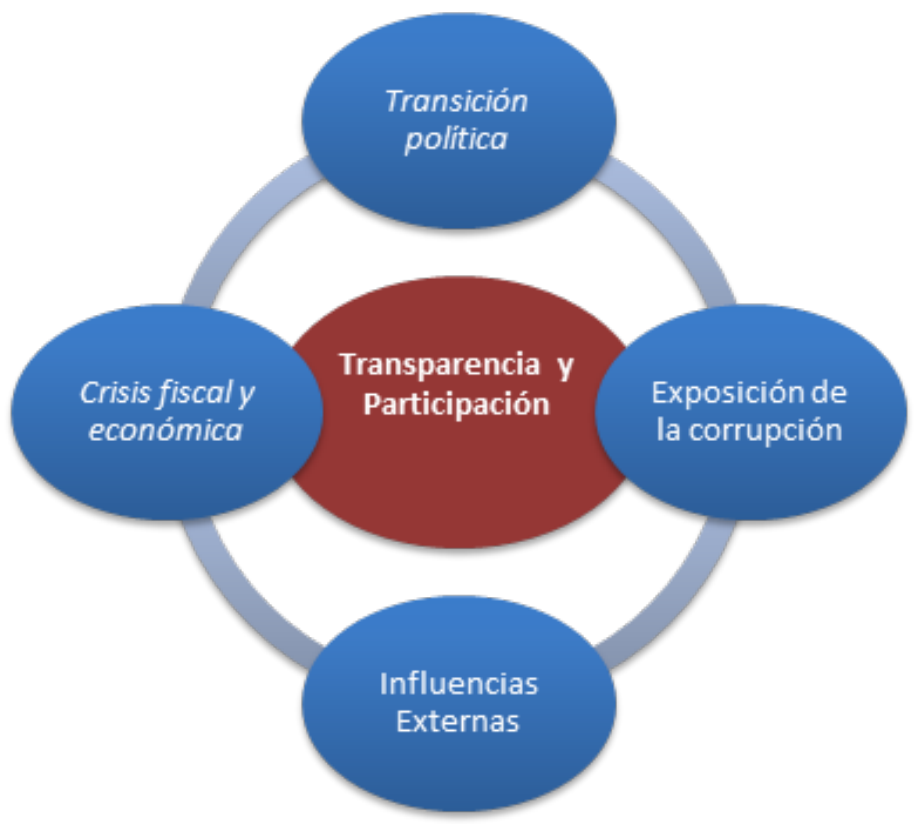

Fuente: Adaptado de Khagram et al. (2013).

La comprensión de estos factores nos lleva a entender las elecciones que fueron hechas por los árbitros políticos y por la sociedad para la construcción de formas de promoción de la transparencia, cambio de los procesos de planificación y mejora del desempeño en el sector público.

De acuerdo con Khagram et al. (2013) estos factores interactúan en combinaciones complejas, para adoptar en los países diferentes formas de interacción de la sociedad con el gobierno y la administración pública, promoviendo o inhibiendo los avances en el proceso de transparencia y participación.

En base a estos cuatro factores principales, Khagram et al (2013) señalaron, por ejemplo, que Brasil estableció mecanismos y capacidades institucionales, a fin de garantizar no sólo la vasta cantidad de información a los ciudadanos, sino también de dar la oportunidad para que diferentes actores, puedan involucrarse en el proceso de planificación presupuestaria.

Es importante resaltar que Brasil vino experimentando cambios en esos cuatros factores, a lo largo de las últimas décadas.

En materia de transición política, Brasil experimentó una significativa transición, que tuvo como marco el régimen militar (1964-1984), pasando por el régimen democrático y el proceso de elecciones indirectas (1984-1989), hasta alcanzar un régimen democrático, con elecciones directas en 1989. Resaltando, que la Constitución de 1988 creó los mecanismos para una mayor implicación de los ciudadanos en el proceso de planificación pública, aunque las condiciones de implantación, no fuesen tan claras.

En relación a las crisis económicas, Brasil tuvo que enfrentarse a un largo periodo de inflación y a restricciones presupuestarias que se intensificaron entre la década de los ochenta y el inicio de los noventa.

En 1993, Brasil experimentó un gran cambio tras un plan económico de éxito (el Plan Real), que tuvo como objetivo controlar la inflación. Ese factor estimuló también la participación popular, principalmente en lo relativo al acompañamiento de los gastos públicos.

La corrupción, tercer factor, es bien conocida en América Latina, como un todo. En los últimos años, especialmente después de 2003, Brasil pasó por una serie de escándalos de corrupción, involucrando a funcionarios federales, funcionarios públicos, políticos y también corporaciones. Estos factores señalaban un problema de gobernanza y desempeño en el sector público, en vista de los efectos de la corrupción sobre los indicadores económicos, sociales y de calidad de la inversión.

En este sentido, cabe indicar que Kaufmann y Bellver (2005) destacan la contribución positiva de la transparencia para la mejora de los indicadores de desarrollo socioeconómico y humano, así como para reducir la corrup- 
ción. Kaufmann y Bellver (2005) añaden que en países con el mismo nivel de renta, el que posee un ambiente más transparente, tiende a tener un mejor desempeño gubernamental, especialmente cuando se trata de prestación de servicios públicos.

Las presiones externas han sido uno de los factores más importantes en los últimos años, primordialmente en 2013, con una sucesión de manifestaciones públicas que tomaron las calles de varias ciudades brasileñas. En esas manifestaciones, la baja calidad del gasto y de los servicios públicos junto a la corrupción fueron algunos de los elementos por los que más se protestó. De ahí, la incontestable importancia de la participación popular en el gobierno, especialmente en el proceso de planificación y control de las políticas públicas.

Hay evidencias de que el país está pasando por una serie de transformaciones en su proceso de planificación e involucración del ciudadano, guiado por la opinión pública y por las presiones de la iniciativa privada y del tercer sector. Ese proceso podría considerarse como una nueva reforma del Estado, inclinada, principalmente, hacia la construcción de nuevos medios de interacción con la sociedad civil, aunque no se sepan los verdaderos propósitos del compromiso popular, siendo esa la base de las reflexiones que se deben realizar.

De acuerdo con Frey (2004:123), dos perspectivas principales han determinado el debate en torno a la reforma y la modernización del Estado y la gestión pública en Brasil, la gerencial y la democrático-participativa.

Para Farah (2004) y Frey (2007) en el modelo gerencial de la nueva administración pública existe una separación entre la esfera política y la esfera administrativa siendo considerada esencial para garantizar resultados y para establecer transparencia y condiciones de accountability. Se observa por tanto un modelo que busca transferir instrumentos de gerencia empresarial al sector público.

Según Frey (2007), en el modelo democrático-participativo, las condiciones de accountability serían impulsadas a través de estímulos a la involucración y a la participación de la sociedad civil organizada y de los ciudadanos en la formulación, implantación y control de las políticas públicas.

El autor destaca también que los distintos modelos tienden a funcionar en sistemas diferentes de implementación y evaluación de las acciones del gestor público, pues, mientras el modelo gerencial pretende aislar y proteger al gestor público de las presiones derivadas de la sociedad y la evaluación se realiza sobre su capacidad de alcanzar las metas establecidas por el sistema de decisión política, el modelo democrático-participativo requiere nuevas habilidades del gestor público, principalmente en la relación con los más variados actores políticos y sociales, ganando importancia en este modelo el proceso en sí de la gestión pública, particularmente en lo referente a su carácter democrático.

Por tanto, se observa que mientras que el modelo gerencial resalta la necesidad de reducción del Estado y la transferencia de instrumentos de gerencia empresarial al sector público, el modelo democrático-participativo destaca la organización de la sociedad civil y la reestructuración de los mecanismos de decisión, a favor de una mayor involucración de la población en el control social de la administración pública y en la definición e implantación de políticas públicas.

Farah (2004) defiende que un híbrido de innovación en la gestión pública, incluyendo aspectos gerenciales y democráticos, llevaría al Estado a presentar incrementos relevantes en el desarrollo de las políticas públicas.

Dentro de la perspectiva democrático-participativa, en la que la participación social es considerada como una herramienta importante para que se dé la buena gobernanza, Young (2006) destaca su importancia para que la gestión sea de hecho democrática. La autora argumenta, también, que una sociedad que posee foros organizados por el Estado y fomentados por la sociedad civil, donde transcurren las discusiones sobre políticas y que influyen en procedimientos y decisiones gubernamentales, es más plenamente democrática.

En este sentido, Souza (2004) se muestra, en su estudio, preocupado con la calidad de la participación en los procesos de formulación e implantación de políticas públicas, buscando establecer medidas para esa participación a partir de adaptaciones de las medidas propuestas por Arnstein (1969).

La escala propuesta por Arnstein (1969), representada en la Figura 2, fue definida en tres niveles: no-participación, participación simbólica o pseudoparticipación y, poder ciudadano. Estas tres categorías, fueron divididas en ocho niveles, representados en el formato de una escalera donde los escalones corresponden en nivel creciente, a medida que se sube cada escalón, a la extensión del poder del ciudadano en decidir las acciones y/o programas. 


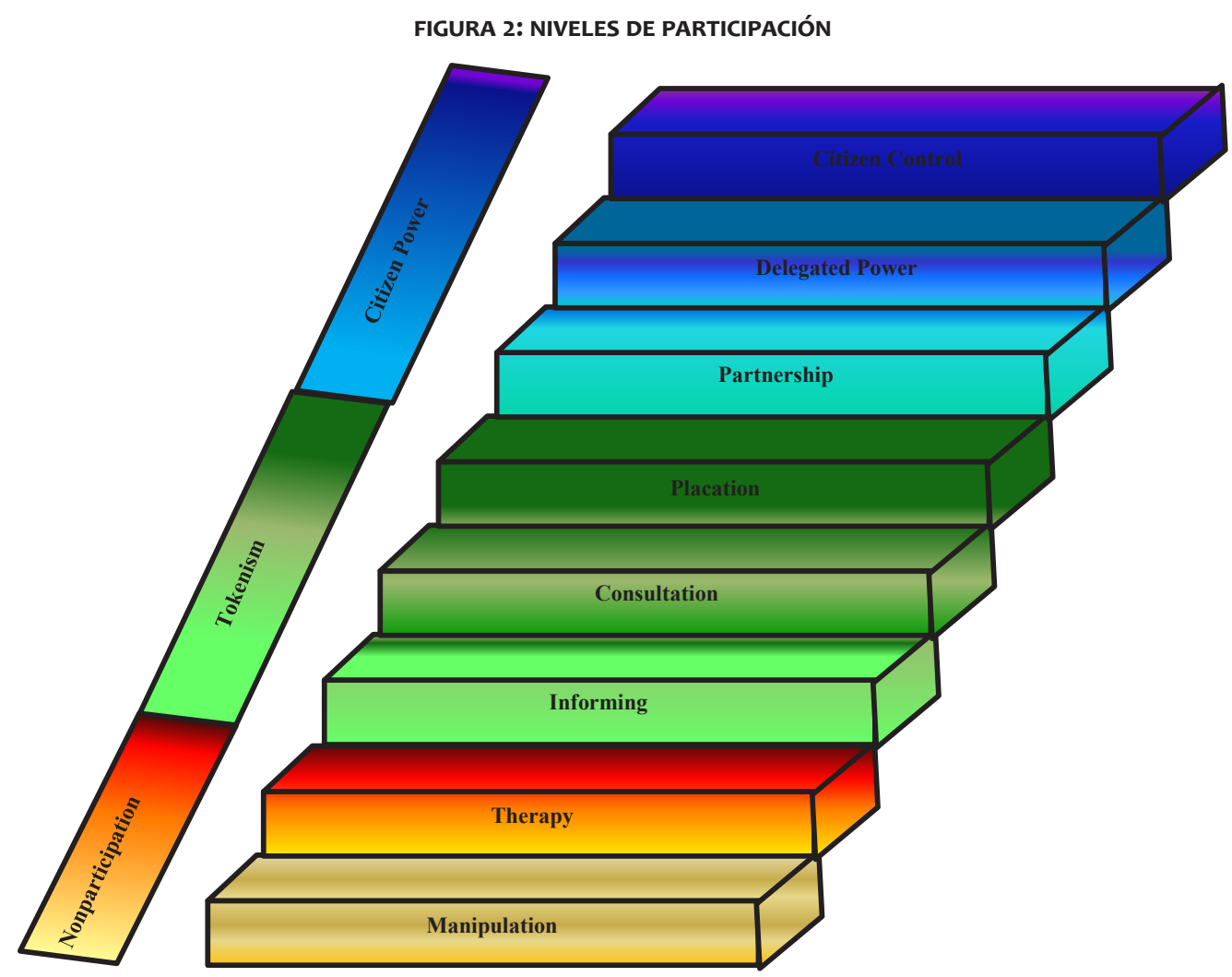

Fuente: Adaptado de Arnstein (1969).

Los dos primeros niveles fueron definidos por Arnstein (1969) como manipulación y terapia, siendo encuadrados en la categoría "no-participación". Su objetivo real es permitir que los que toman las decisiones pudiesen educar o tratar a los participantes. Por tanto, no permiten a la población participar en los procesos de planificación o tomar decisiones en relación a las políticas públicas y programas.

Los tres niveles siguientes fueron denominados información, consulta y pacificación o apaciguamiento, perteneciendo a la categoría Pseudoparticipación. Los niveles información y consulta, permiten una participación limitada. Es decir, los que antes no tenían derecho a participar pueden, ahora, escuchar y ser escuchados. En estos dos niveles, los ciudadanos no tienen poder para asegurar que sus opiniones serán aceptadas por los titulares del poder. En el nivel pacificación, la limitación continúa, los ciudadanos pueden aconsejar a los “poderosos”, aunque son estos quienes tomarán la decisión.

Los tres últimos niveles, definidos como colaboración, poder delegado y control ciudadano, son los que permiten al ciudadano obtener una participación efectiva, es decir, alcanzar el llamado por Arnstein (1969) "poder ciudadano". En el nivel colaboración los ciudadanos participan en colaboración, pudiendo negociar, al mismo nivel, con los que detentan el poder. En los niveles poder delegado y control ciudadano, los ciudadanos son mayoría en los foros de toma de decisiones, poseyendo el completo poder gerencial.

Souza (2004) efectúa algunas adaptaciones en la escala propuesta por Arnstein (1969), creando una nueva escala de evaluación de los procesos decisorios participativos de planificación y gestión, que va desde la participación auténtica a la no-participación (Figura 3). 

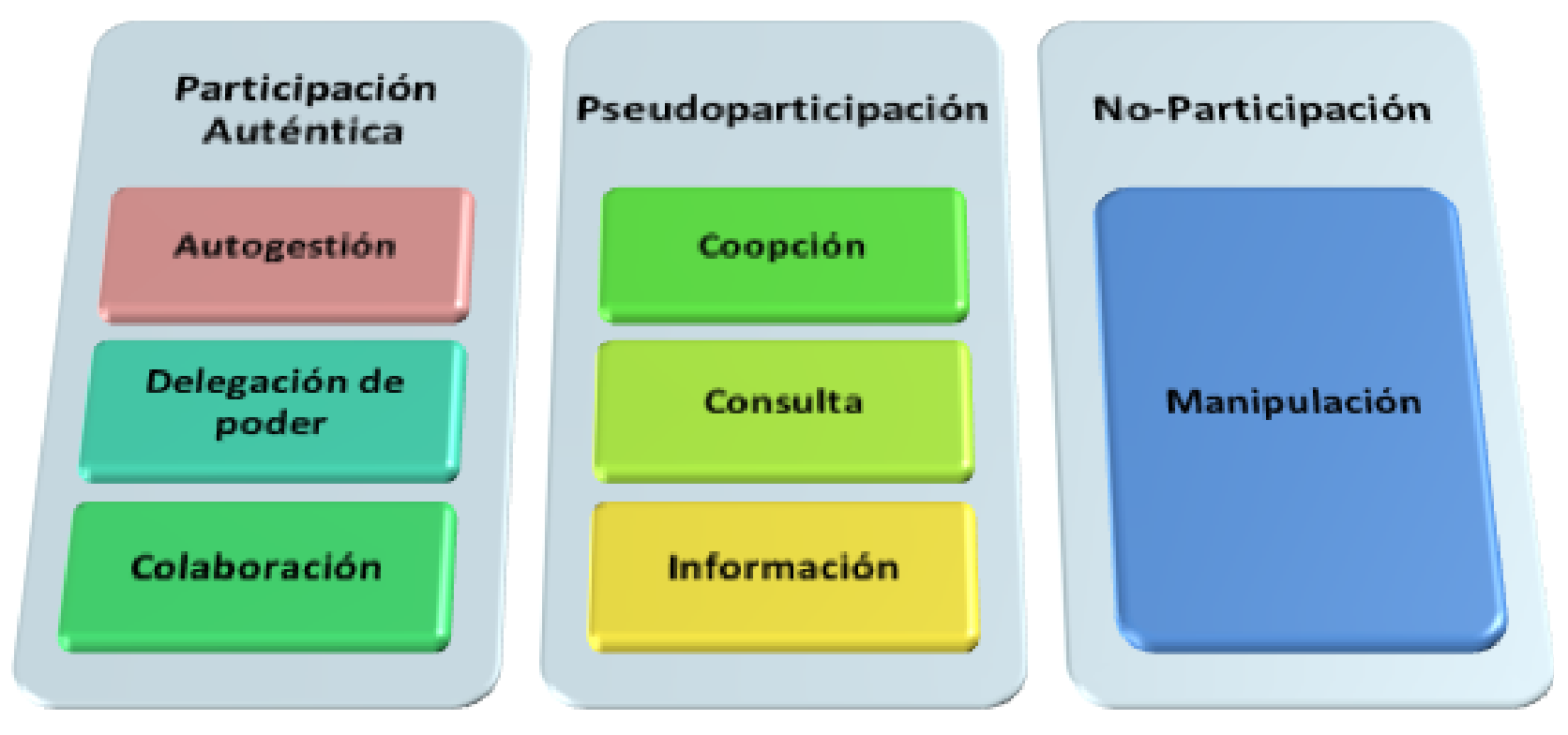

Fuente: Adaptado de Souza (2004).

La propuesta de Souza (2004) abarca entonces, tres grados de participación, a saber: 1) No-participación; 2) Pseudoparticipación y 3) Participación Auténtica, que varían de acuerdo con siete categorías (manipulación, información, cooptación, colaboración, delegación de poder y autogestión).

En la categoría manipulación está englobado el nivel terapia propuesto por Arnstein (1969), que puede ser considerado una variante de manipulación, en la cual no encajarían formas más duras de manipulación, para las cuales Souza (2004) propone su sustitución por la situación de coerción. En esta situación no habría participación, la población involucrada sería inducida a aceptar políticas e intervenciones, perteneciendo al grado de no-participación.

En la categoría de información, las informaciones sobre las políticas e intervenciones son puestas a disposición de la población. En la categoría consulta la población es interrogada sobre políticas e intervenciones por medio de encuestas de opinión, audiencias públicas o similares. La categoría cooptación correspondería al nivel pacificación propuesto por Arnstein (1969). En esta categoría la población es cooptada (consultada, escuchada sistemáticamente) en relación a políticas e intervenciones, a través de canales e instancias permanentes de participación. Esas tres categorías serían pertenecientes al grado de Pseudoparticipación, por tratarse de una participación considerada aún limitada en relación a la planificación e implantación de políticas públicas.

Por último, estarían situadas las tres categorías, donde según Souza (2004), se alcanzaría el grado de participación auténtica o participación efectiva. Estas tres categorías serían colaboración, delegación de poder y autogestión. La categoría de autogestión correspondería al nivel entendido como “control ciudadano". En esta categoría, las políticas e intervenciones son conducidas de modo autogestionario por la población como sociedad civil, con la total ausencia del Estado. En la categoría delegación del poder, el Estado abdica de atribuciones, relativas a políticas e intervenciones, en favor de la población, como sociedad civil, actuando en cogestión con la misma. Ya en la categoría colaboración, la sociedad civil y el Estado colaboran en políticas e intervenciones, dividiendo responsabilidades, aunque mantengan sus atribuciones formales.

La aplicación de este modelo en la comprensión de la involucración del ciudadano en el proceso de planificación abre la perspectiva para la comprensión de nuevas formas de gobernanza en el sector público, así como 
permite evaluar la calidad de esa interacción.

\section{LA GOBERNANZA Y LA PLANIFICACIÓN EN EL SECTOR PÚBLICO}

El término "Gobernanza corporativa" tiene su origen en el sector privado y en sus concepciones tradicionalmente enfocadas a la relación empresa-accionistas (Lethbridge, 1997). Sin embargo, cada vez más, definiciones de gobernanza corporativa han enfatizado en un conjunto más amplio de relaciones para incluir aquellas involucradas en la organización o afectadas por la misma, como funcionarios, directores, proveedores, accionistas y partes interesadas, clientes y comunidades locales, los stakeholders.

En el proceso de planificación diversos autores han destacado los diseños de gobernanza en el sector público, como Timmers (2000) y Barret (2005). Hay evidencias en la literatura sobre los efectos positivos de la transparencia sobre la Gobernanza en el sector público (ISLAM, 2003).

La Organization for Economic Co-Operation and Development - OCDE (2004:11) tiene una visión amplia de la gobernanza corporativa y la define como el conjunto de relaciones entre la gestión de una empresa, su consejo de administración, sus accionistas y otras partes interesadas. Esta proporciona la estructura a través de la cual son definidos los objetivos de la empresa y los medios para alcanzar los objetivos y controlar el desempeño. De ahí el propósito de discutir gobernanza en el proceso de planificación en el sector público.

Es decir, por ser la transparencia parte importante del proceso de planificación, el ciudadano debe ejecutar un doble papel, ser protagonista y beneficiario de las acciones del Estado. Sin embargo, para que eso ocurra debe tenerse en mente la construcción de un nuevo modelo de gobernanza que sostenga la planificación multiprotagonizada, conforme a lo esbozado en la Figura 4.

FIGURA 4: MODELO DE PLANIFICACIÓN MULTIPROTAGONIZADA EN EL SECTOR PÚBLICO

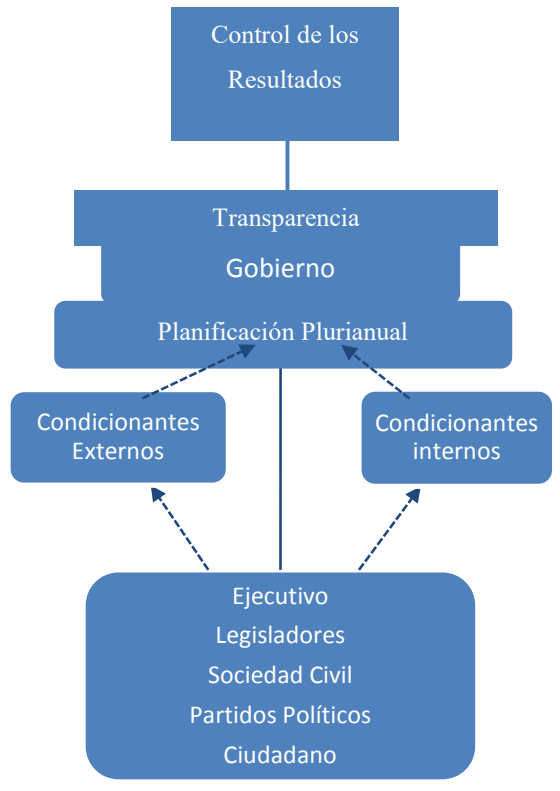

Fuente: Elaborada por los autores.

Para Matias-Pereira (2010) la gobernanza en las organizaciones públicas y privadas presenta significativas similitudes como: la separación entre propiedad y gestión, los instrumentos definidores de responsabilidades y poder y el acompañamiento e incentivo en la ejecución de las políticas y objetivos definidos, entre otros. El autor destaca también que la gobernanza en el sector público está orientada hacia las formas de interacción en las relaciones de poder entre el Estado, el gobierno y la sociedad, dedicando especial atención a los procesos de cooperación y a los mecanismos para estimular las relaciones entre los principales actores involucrados en este esfuerzo, el gobierno y los stakeholders (sector privado, tercer sector y sociedad).

Basándonos en este enfoque, se pueden comprender las iniciativas de planificación como proceso de ejecución del gobierno, pero que se apoya, principalmente en el compromiso social y en el entrelazado de relaciones 
entre diferentes actores, cuya formulación y ejecución dependen también de un conjunto de factores externos e internos.

Algunos de estos factores son ampliamente discutidos en la literatura, por un número creciente de trabajos, aunque pocos de ellos dediquen atención a los desafíos de la implantación de la planificación plurianual como forma de promoción de una nueva estructura de gobernanza comprometida con resultados a largo plazo. No siendo tampoco identificados trabajos que exploren el PPA más allá de las vertientes formales de la planificación.

Ese proceso gana mayor dimensión cuando le es atribuido el carácter de transparencia y un proceso de accountability frutos de la gobernanza pública. Timmers (2000) presenta cuatro elementos en ese proceso de gobernanza, a saber: la Administración (realizar objetivos por medio de la estructuración y desarrollo de procesos); el Control (sistema de medidas y procedimientos implantados y mantenidos); la Supervisión (garantizando que los objetivos de la organización lo sean en beneficio de la sociedad) y; Accountability (deber de proporcionar información sobre todas las tareas y poderes delegados).

Respecto a los resultados deseados en el sector público, la gobernanza aspira a la mejora de los procesos y resultados y al compromiso de los actores.

\section{METODOLOGÍA}

El esquema metodológico adoptado en esta investigación está presentado en dos partes, siendo estas: a) Fuente de datos y periodo de investigación y b) Procedimientos metodológicos.

Como fuente de datos fueron utilizados diferentes instrumentos de recogida de datos en Ciencias Sociales Aplicadas, los cuales serán descritos en este trabajo.

El análisis incluyó, esencialmente, la producción de un estudio de caso sobre la participación popular en la Planificación Pública del Gobierno de Minas Gerais (PPAG) durante el periodo 2012-2015.

\section{FUENTE DE DATOS Y PERIODO DE LA INVESTIGACIÓN}

La investigación se realizó basándonos en tres fuentes de datos fundamentales: documentos oficiales, observación participante y entrevistas. Como fuentes documentales fueron utilizados el Plan de Acción Gubernamental 2012-2015, e informaciones públicas disponibles en las páginas web de la Secretaria de Estado de Planificación y Gestión de Minas Gerais y de la Asamblea Legislativa de Minas Gerais referentes a la planificación estatal.

En este estudio fueron utilizados diferentes tipos de entrevista, siguiendo la nomenclatura propuesta por Marconi y Lakatos (2009). La entrevista estandarizada o estructurada en que se sigue un guion, en la que el entrevistador ya posee preguntas previamente definidas. Y la entrevista no estructurada, sin guion modelo, donde el investigador puede dirigir cada situación de la forma que considere más adecuada, permitiendo explorar las cuestiones de una forma más extensa.

Las entrevistas fueron realizadas a actores clave en la planificación en Minas Gerais, incluyendo a la Secretaria de Estado de Planificación y Gestión de Minas Gerais, Legisladores mineros, Representante de la Comisión de Participación Popular y la Consultoría de la Asamblea Legislativa de Minas Gerais.

Los actores entrevistados fueron (i) Subsecretario de Planificación, Presupuesto y Calidad del Gasto; (ii) Representante de la Subsecretaría de Gestión de la Estrategia Gubernamental; (iii) Consultora de Finanzas y Presupuesto de la Asamblea Legislativa; (iv) Consultora de Acompañamiento y Evaluación de Políticas Públicas de la Asamblea Legislativa; (v) Presidente de la Comisión de Participación Popular de la Asamblea Legislativa; (vi) Director de Procesos Legislativos; (vii) Diputados pertenecientes y no pertenecientes a la base aliada del Gobierno.

Fue utilizada también, la técnica de observación participante, mediante la presencia en una audiencia pública con participación popular, pretendiendo captar el proceso de conducción de las actividades de control, comunicación de las metas y resultados y de promoción del compromiso popular.

La Figura 5 resume las estrategias de recogida de datos, esbozando el público y los instrumentos utilizados en las diferentes etapas de la investigación. 
FIGURA 5: TÉCNICAS DE RECOGIDA DE DATOS

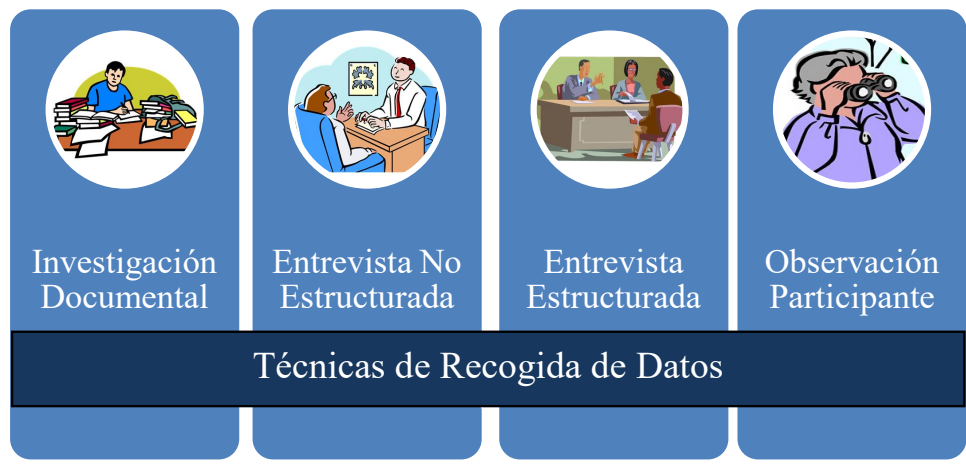

Fuente: Elaborada por los autores.

\section{ESTUDIO DE CASO}

El estudio de caso fue escogido como técnica metodológica central de este trabajo. El estudio de caso es una técnica de investigación empírica que busca explicar fenómenos contemporáneos dentro de un contexto determinado, principalmente si los límites entre el fenómeno y el contexto no están debidamente definidos (Yin, 2001:32). Según Gil (2010:51) el estudio de caso tiene como característica el estudio profundo y exhaustivo de un determinado objeto con la intención de permitir su conocimiento de manera amplia y detallada.

La producción del estudio de caso tuvo como foco el proceso de participación popular en el proceso de gestión en el Estado de Minas Gerais.

\section{RESULTADOS Y DISCUSIONES}

La Asamblea Legislativa de Minas Gerais desde el inicio de la década de los noventa procura desarrollar actividades que promuevan el debate entre diversos segmentos de la sociedad sobre aspectos de la planificación en el ámbito estatal. Aunque la regionalización aún sea incipiente, Fleury (2009) destaca que esas actividades abarcan desde grandes eventos institucionales como seminarios legislativos, ciclo de debates y Foros hasta visitas y audiencias públicas promovidas por las comisiones permanentes tanto en la capital como en el interior.

La Resolución n5.117, aprobada en 1992, abordaba específicamente el proceso de las audiencias públicas en el Estado. En 1997 la participación de la sociedad tenía un reconocimiento institucional y la ampliación del proceso es objeto específico del Título VIII del reglamento interno de la Asamblea Legislativa (FLEURY, 2009).

Entre los años 1996-1998 la discusión en la Asamblea Legislativa era en torno a la naturaleza de la participación popular. Fleury (2009) destaca que a finales del año 1996 se tramitó un proyecto de ley que "confería a las entidades populares implicadas en el proceso presupuestario la competencia para definir, dentro de los límites prefijados por el Ejecutivo, las acciones y obras a ser ejecutadas". Se buscaba de esa manera una democracia menos centralizada y más participativa.

Después de muchas discusiones el proyecto fue aprobado con sus propuestas originales asegurando que la participación popular tendría naturaleza deliberativa. Sin embargo, Fleury (2009) destaca que la aprobación no garantizó la eficacia del proceso de elaboración participativa del presupuesto.

En 1999, primer año de gobierno de Itamar Franco, fueron realizadas audiencias públicas de planificación en todas las regiones del Estado. No obstante, Fleury (2009) apunta que como las propuestas definidas, incluidas en el presupuesto estatal no fueron ejecutadas plenamente, la asamblea acabó por suspender el proceso en los años siguientes.

Después de ese episodio la participación popular vuelve a tener relevancia en la asamblea, con la creación en 2003, durante el primer año del gobierno Aécio Neves, de la Comisión de Participación Popular que abría la posibilidad de intervención de la sociedad civil, junto a la Asamblea Legislativa en la elaboración de la planificación y presupuesto estatal.

Más tarde, a partir de 2003, se inaugura la Asamblea Legislativa do Estado de Minas Gerais - ALMG, a través de la Comisión de Participación Popular en colaboración con la Secretaría de Estado de Planificación y Gestión, el 
proceso de revisión popular del PPAG, es decir, el proceso de discusión popular del Plan Plurianual de Acción Gubernamental (PPAG), con la realización de audiencias públicas específicas para la presentación de propuestas de enmienda a los programas y proyectos que componen el PPAG.

Se observa entonces, el esfuerzo por implantar una forma de gestión pública democrático-participativa en el Estado de Minas Gerais, con el modelo de lo que Frey (2007) definió como democrático-participativo, en el que las condiciones de accountability serían fomentadas a través de la realización de estímulos a la implicación y participación de la sociedad civil organizada y de los ciudadanos en la formulación, implantación y control de las políticas públicas.

Dentro de la perspectiva democrático-participativa, en la que la participación social es considerada como una herramienta importante para que la buena gobernanza ocurra, fueron analizados los estímulos a la participación popular utilizados por órganos del ejecutivo y del legislativo del Estado.

Sin embargo, actores que influyen en la planificación en el ámbito del poder ejecutivo declararon que la Secretaría de Estado de Planificación y Gestión (SEPLAG) del Estado de Minas Gerais no posee canales institucionales que permitan la intervención efectiva de la sociedad civil en el proceso de elaboración, control y evaluación del PPAG. Aun así, existe un proyecto piloto que ha sido desarrollado por la SEPLAG, donde fueron realizados foros regionales en dos de las diez regiones administrativas, lo que demuestra que el poder ejecutivo se ha preocupado de crear canales de participación de la sociedad civil.

El canal de participación de la sociedad civil que presenta mayor efectividad y que tiene mayor relevancia es el desarrollado por la Asamblea Legislativa del Estado de Minas Gerais, a través de la Comisión de Participación Popular.

La Comisión de Participación Popular (CPP) fue creada en junio de 2003, durante el primer gobierno de Aécio Neves, en un intento de ampliar la participación directa del ciudadano en los trabajos legislativos. Corresponde a la comisión recibir y apreciar proposiciones procedentes de la sociedad que puede, incluso, presentar proyectos de ley y enmiendas a proyectos de ley, además de solicitar audiencias públicas y otras actividades legislativas.

La CPP es una comisión permanente de la Asamblea Legislativa y su creación fue inspirada en la Comisión de Legislación Participativa de la Cámara de los Diputados, por ello, posee un funcionamiento similar a la misma.

La sociedad civil puede presentar a la CPP propuestas de corrección de los trabajos legislativos y proposiciones de acciones legislativas tales como enmiendas a proyectos de ley en tramitación, proyectos de ley, solicitud de audiencia pública y consulta pública.

La CPP ofrece nuevas posibilidades de participación a la sociedad civil siendo un instrumento más del Legislativo minero para fortalecer la democracia. Se trata de un instrumento que debe ser institucionalizado, siendo necesaria una colaboración con el gobierno del Estado.

La Comisión de Participación Popular tiene la función de recoger propuestas de la sociedad para que sean analizadas y, si procede, ser incorporadas al PPAG. Cabe resaltar que la participación de la sociedad civil puede darse a través de propuesta directa enviada a la Comisión de Participación Popular, y/o en las audiencias públicas de monitorización y/o en las de revisión del PPAG.

A partir de 2009, debido a la aprobación de la enmienda al proyecto de ley de revisión del PPAG 2008-2011, para el ejercicio 2009, la Asamblea junto al Gobierno, vienen debatiendo con la sociedad la monitorización de la planificación del Estado.

En las audiencias públicas de control, que ocurren normalmente en junio, es presentada la ejecución física y financiera de las acciones que están siendo implantadas a través de los programas del gobierno para que la sociedad civil acompañe la ejecución de las políticas públicas. Al final de cada presentación, el micrófono queda abierto a todos para cualquier cuestión referente a la ejecución.

En el año 2012 hubo observación participante durante las audiencias de control, en un intento de percibir cómo ocurre el proceso y si hay participación directa de la sociedad civil y sus representantes en las audiencias.

Se apreció que hay participación efectiva, de la sociedad civil, durante el control de la ejecución de los programas del PPAG, una vez que representantes de entidades relacionadas a las temáticas analizadas estaban presentes y participaron de las discusiones.

Además de asegurada la participación popular en las audiencias de control, la misma es garantizada también en las audiencias de revisión del PPAG que ocurren entre los meses de octubre y noviembre de cada año. 
La propuesta del PPAG es acordada por la SEPLAG, hasta el día 30 de septiembre de cada año y enviada a la asamblea. Una vez recibida la propuesta, la ALMG efectúa el análisis y construye una sistemática de discusión, que desde 2011 es realizada en las comisiones de la propia asamblea, contando con la participación creciente de los diputados, y a partir de ahí el PPAG es discutido junto a la sociedad civil en las audiencias de revisión.

Durante las audiencias púbicas de revisión, la población puede presentar propuestas de enmienda al PPAG y después de esas audiencias, la SEPLAG se reúne con los consultores de la asamblea, con los representantes de las áreas temáticas y con los diputados que tengan interés y quieran participar de ese proceso. En esa reunión son discutidas esas propuestas, no en el sentido de vetarlas, sino de evaluar su viabilidad como enmiendas al PPAG.

Las audiencias de revisión del PPAG, en 2012, se realizaron entre los meses de octubre y noviembre en el interior y en la capital del estado. Las audiencias públicas fueron realizadas divididas por áreas temáticas, según las redes de desarrollo integrado propuestas en el plan.

Cabe destacar que en la revisión del PPAG 2012-2015, en el ejercicio 2013, fueron presentadas, entre octubre y noviembre, 460 propuestas de iniciativa popular en las audiencias públicas. Esas sugerencias dieron lugar a 195 propuestas de acción legislativa (PLEs), que se convirtieron en 80 enmiendas al PPAG y 73 enmiendas a la Ley Presupuestaria, con un impacto financiero de 13 millones.

La Figura 6 ilustra la participación popular en la proposición de enmiendas así como la adhesión por parte del ejecutivo a dichas enmiendas.

\section{FIGURA 6: PARTICIPACIÓN POPULAR EN LA PROPOSICIÓN DE ENMIENDAS Y ADHESIÓN DEL EJECUTIVO}

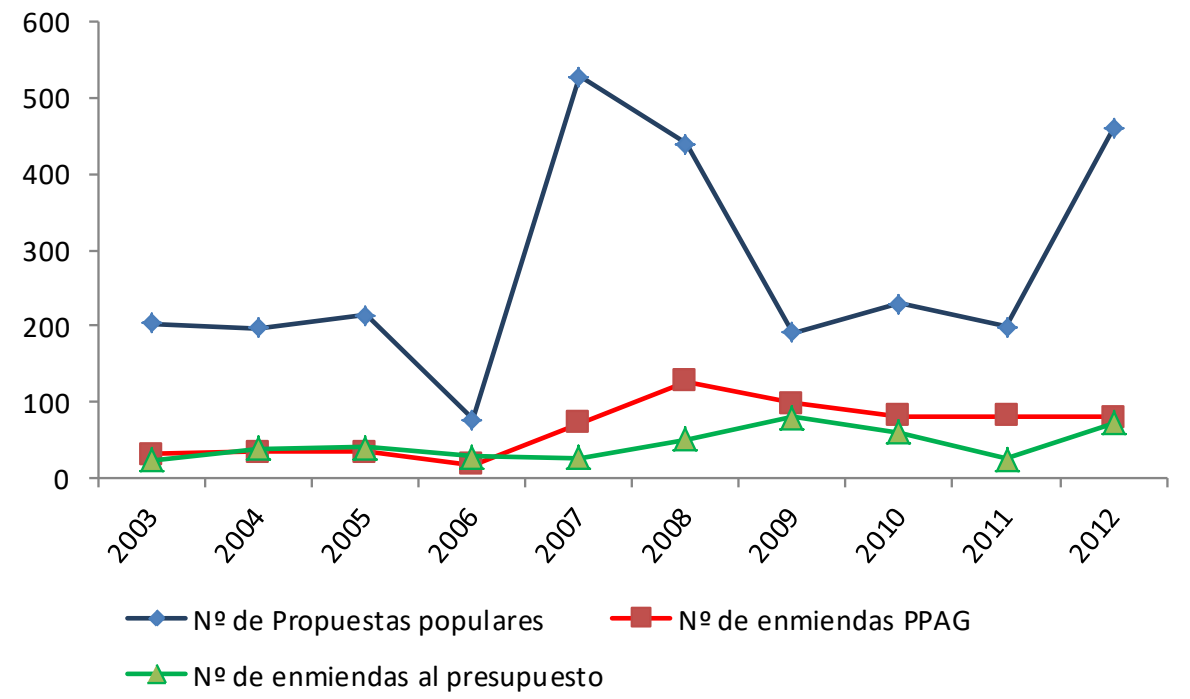

Fuente: Elaborada por los autores.

Se observa que la sociedad civil ha propuesto innumerables enmiendas y que el volumen aceptado es considerable, dado que la aceptación o no de una enmienda depende de diversos factores, que van desde la voluntad gubernamental o la disponibilidad presupuestaria hasta la pertinencia de la propuesta.

Como se resaltó anteriormente la Asamblea, buscando mayor participación popular, ha realizado audiencias regionalizadas, para discutir la revisión del PPAG, en las 10 regiones administrativas. La elección de los municipios se produce generalmente en función del tamaño de la ciudad y de la indicación de los parlamentarios, por eso generalmente estas audiencias acontecen en ciudades-centrales. En estas audiencias, las entidades representativas de la sociedad civil presentan propuestas, que serán analizadas por la ALMG y si fuera posible serán adicionadas al PPAG y al presupuesto.

Cada propuesta es analizada y traducida como pieza presupuestaria por el cuerpo técnico de la ALMG y enviada a la CPP. En el caso de que sea juzgada como pertinente por la Comisión de Participación Popular, la misma es presentada como enmienda en el formato que fue sugerido por el cuerpo técnico de la ALMG. Tras el análisis de la Comisión de Fiscalización Financiera, esa enmienda es presentada al proyecto de ley de revisión del PPAG como las enmiendas de los demás parlamentarios. 
La realización de audiencias y foros viene a reforzar la gestión democrática pues de acuerdo con Young (2006) una sociedad que posee foros organizados por el estado y fomentados por la sociedad civil, donde transcurren las discusiones sobre políticas, y que influyen en los procedimientos y decisiones gubernamentales, es más plenamente democrática.

A medida que esos foros generan discusiones, por medio de la participación de la sociedad civil en el PPA, que alteran en el ejecutivo el proceso de planificación, se da un proceso de innovación en la relación entre sociedad civil y gobierno.

Hay que destacar que esos avances coincidieron con la implantación, por el gobierno estatal de Minas Gerais, de un modelo gerencial basado en incentivos, contratos de objetivos y desempeño, conocido como "choque de gestión".

Esos avances podrían apuntar a la implantación del modelo híbrido preconizado por Farah (2004), basado en aspectos gerenciales y democráticos, con impacto sobre la calidad de las políticas públicas.

Por otro lado, la participación no es todo. Es importante también analizar cuál es el nivel de calidad de la participación en procesos de formulación e implantación de políticas públicas.

En el Estado de Minas Gerais la información relativa a la planificación es puesta a disposición de la población a través de portales tanto del legislativo como del ejecutivo.

Los informes de control son emitidos bimestralmente, siendo producidos seis informes institucionales de control del PPAG anualmente, que organizan la información bimestralmente, de forma acumulativa. Así, el último informe presenta información sobre la ejecución de objetivos físicos y financieros de todo el ejercicio. Esos informes están disponibles en la página web de la SEPLAG. En dicha página, se encuentra disponible información sobre el número de enmiendas propuestas e incorporadas al PPAG.

Se observa en este caso, que la participación se encuentra en la categoría denominada por Souza (2004) “información”, en la que la información sobre las políticas e intervenciones es puesta a disposición de la población.

La realización de audiencias de control y revisión y foros, por el legislativo y el ejecutivo, donde la población es interrogada sobre políticas e intervenciones demuestra la existencia de la categoría de participación "consulta".

La creación de la Comisión de Participación Popular, que puede ser considerada un canal permanente de participación, que permite a la población ser consultada y escuchada sistemáticamente, representa la categoría "cooptación".

Se observa entonces, que la participación popular en el estado analizado se encuentra en el nivel dos de la escala propuesta por Arnstein (1969) y adaptada por Souza (2004), ocurriendo entonces una "Pseudoparticipación", pues las tres categorías encontradas serían pertenecientes al grado de Pseudoparticipación, por tratarse de una participación considerada aún limitada en relación a la planificación e implantación de políticas públicas.

Aunque la participación social en el estado sea encuadrada en el nivel o grado de "pseudoparticipación", se observan pasos en dirección al nivel de "participación auténtica", una vez que el estado ha buscado establecer una colaboración con la sociedad civil, para la elaboración de políticas e intervenciones. Además, se observan trazos indicativos de la categoría "Colaboración" a través de la apertura a propuestas de enmiendas por parte de la sociedad y de la incorporación de esas enmiendas en la planificación.

\section{Consideraciones finales}

Este estudio tuvo como objetivo verificar cómo ha ocurrido y cuál es el nivel de participación popular en los procesos de elaboración, control y evaluación del Plan de Acción Gubernamental en la esfera estadual del gobierno Brasileño.

De entre los principales resultados, destaca el hecho de que el Legislativo ofrezca canales que estimulen la participación popular en el proceso de planificación del estado, principalmente a partir de la creación de la Comisión de Participación Popular (CPP), en el año 2003. En la elaboración de la Planificación Plurianual de Acción del Gobierno (2012-2015), hubo también un esfuerzo del ejecutivo, de inserción de la sociedad civil, a través de la realización de audiencias en dos regiones del estado, donde los participantes pudieran expresar las prioridades de la región. 
Por tanto, la participación popular se ha producido a través de diversos canales, como: audiencias públicas efectuadas por el ejecutivo en las regiones de planificación; propuestas directas de la sociedad a la Comisión de Participación Popular; y, audiencias públicas de control y/o de revisión del Plan Plurianual de Acción Gubernamental.

Se observa que el nivel de participación apunta en dirección a la "participación auténtica" en el Estado de Minas Gerais, puesto que se observa la existencia de la categoría de participación auténtica "Colaboración".

Ante la naturaleza representativa del gobierno brasileño, parece posible apenas el alcance del grado de "participación auténtica” de las categorías “colaboración” y “poder delegado”, siendo la "autogestión” un ideal utópico para el nivel de desarrollo de la democracia brasileña.

Sin embargo, es oportuno registrar que ha ocurrido en la gestión pública del estado de Minas Gerais una legitimación o incluso una institucionalización de la participación popular que, aunque pueda ser vista como un punto positivo carece de la mayor implicación del ciudadano, una vez que la mayor implicación sistemática es de entidades representativas de la sociedad civil. Tal práctica de participación no afecta a la legitimidad del proceso, pero suscita cuestiones sobre la calidad pública de los intereses defendidos, lo que abre espacio para nuevas cuestiones, con implicaciones en la propagación de conocimientos acumulados en la literatura internacional del área.

\section{BIBLIOGRAFÍA}

ARNSTEIN, S. R. (1969): "The ladder of citizen participation", Journal of the American Institute of Planners, Boston, v.3, n.4, pags.216-224.

BARRET, P. (2005): Achieving better practice corporate governance in the public sector. AM Auditor General for Australia. Disponible en: <http://www.anao.gov.au>. Acceso: 20 jun. 2012.

BOVENS, M. (1998): The quest for responsibility: Accountability and citizenship in complex organisations. Cambridge University Press.

BRESSER-PEREIRA, L.C. (1998): “Gestão do setor público: estratégia e estrutura para um novo Estado", en BRESSER PEREIRA, L.C.; SPINK, P. Reforma do Estado e administração pública gerencial. Rio de Janeiro: FGV, pags. 21-38.

COSTA, L. F. L. G.; CAMELO, G. L. P. (2010): “Gestão Pública Participativa: Aspectos de Governabilidade e Interação Político-Social”, HOLOS, Ano 26, Vol 2., pags.131-141.

DEVAS, N., y GRANT, U. (2003): “Local government decision-making—citizen participation and local accountability: some evidence from Kenya and Uganda”, Public Administration and Development, 23(4), pags. $307-316$.

FADUL, E. y MAC-ALLISTER, M. (2007): “Participação, Governabilidade e Governança Nos Processo Decisórios das Conferências das Cidades”, en XII Encontro Da Associação Nacional De Pós-Graduação e Pesquisa em Planejamento Urbano e Regional. Belém, Pará, 21 a 25 de mayo.

FARAH, M. F. S. (2004): “Inovação e Governo Local do Brasil Contemporâneo” en JACOBI, P.; PINHEIRO, J. A. Inovação do Campo da Gestão Publica Local: Novos desafios, novos patamares. Rio de Janeiro: FGV Editora, pags. 42-75.

FLEURY, S. J. F. (2009): “A participação da sociedade no planejamento estadual: avanços e desafios” en OLIVEIRA, Myriam Costa de (Org). A Assembleia de Minas e a construção coletiva de políticas públicas: eventos institucionais. Belo Horizonte: Assembleia Legislativa do Estado de Minas Gerais.

FREY, K. (2004): “Governança interativa: uma concepção para compreender a gestão pública participativa?”, Revista Política \& Sociedade, n. 5, pags. 117-136, octubre.

FREY, K. (2007): “ Governança Urbana e Participação Pública”, RAC-Eletrônica, v. 1, n. 1, p. 136-150, Jan./Abr.

GIL, A. C. (2010): Métodos e Técnicas de Pesquisa Social. 6. ed. São Paulo: Atlas.

GOLDFRANK, B. (2006): “Lessons from Latin American Experience in Participatory Budgeting”, Presentation at the Latin American Studies Association Meeting, San Juan, Puerto Rico, March 2006.

GRIMMELIKHUIJSEN, S. (2012): "A good man but a bad wizard. About the limits and future of transparency of democratic governments", Information Polity: The International Journal Of Government \& Democracy In The Information Age, 17(3/4), pags. 293-302. 
ISLAM, R. (2003): “Do More Transparent Governments Govern Better?”, World Bank Policy Research Working Paper 3077, Washington DC: World Bank.

KAUFMANN, D. y BELLVER, A. (2005): “Transparenting Transparency: Initial Empirics and Policy Applications”, World Bank Institute Working Paper, Washington DC: World Bank.

KHAGRAM, S.; FUNG, A. DE RENZIO, P. (2013): Open Budgets: The Political Economy of Transparency, Participation, and Accountability. Washington, D.C. Brookings Inst Pr. 264 P .

KOLSTAD, I. AND WIIG, A. (2009): "Is Transparency the Key to Reducing Corruption in Resource-Rich Countries?”, World Development 37 (3), pags. 521-532.

LETHBRIDGE, Eric (1997): “Governança Corporativa”, Revista BNDES, n.8, dezembro.

MARCONI, M. A., LAKATOS, E. M. (2009): Fundamentos de Metodologia Científica. 6 ed. São Paulo: Atlas.

MATIAS-PEREIRA, J. (2010): A governança corporativa aplicada no setor público brasileiro. APGS, Viçosa, v.2, n.1, pags. 109-134, jan./mar.

MILANI, C. R. S. (2008): “O princípio da participação social na gestão de políticas públicas locais: uma análise de experiências latino-americanas e europeias”, RAP - Rio de Janeiro,42(3), pags. 551-579, maio/junho.

MINAS GERAIS (1992): Resolução n 5117, de 13 de julho de 1992. Dispõe sobre normas complementares ao Regimento Interno para a realização de Audiências Públicas Regionais de Comissão Permanente da Assembleia Legislativa. Belo Horizonte, 1992. Disponible en: < http://hera.almg.gov.br/cgi-bin/nph-brs?d=NJMG\&u=http://www. almg.gov.br/njmg/chama_pesquisa.asp\&SECT7=LINKON\&p=1\&r=1\&l=1\&f=G\&s1=resolucao+5117+1992.norm. $>$. ACceso: 12 enero 2013.

ORGANISATION FOR ECONOMIC CO-OPERATION AND DEVELOPMENT - OECD (2004): OCDE Principles of Corporate Governance. Disponible en: http://www.oecd.org/corporate/corporateaffairs/corporategovernanceprinciples/31557724.pdf. Acceso: 20 mayo 2012.

OVERSEAS DEVELOPMENT INSTITUTE (ODI) (2007): “Budget monitoring and policy influence”. Briefing Paper 16, London. 3 p.

PRAT, A. (2005): “The wrong kind of transparency”, American Economic Review 95 (3), pags. 862-877.

PREMCHAND, A. (2001): "Fiscal Transparency and Accountability, Idea and Reality", Paper prepared for the workshop on Financial Management and Accountability, Rome.

SOUZA, M. L. de (2004): Mudar a Cidade: uma introdução crítica ao planejamento e à gestão urbanos. 3. Ed. Rio de Janeiro: Bertrand Brasil.

TEIXEIRA, A. C., DAGNINO, E. \& ALMEIDA C. C. (2002): “La constitución de la sociedad civil en Brasil” en DAGNINO, E. (org.), Sociedad Civil, Espacios Públicos y Democratización: Brasil. México: Fondo de Cultura Económica, pags. 21-76.

TIMMERS, Hans (2000): Government Governance: Corporate governance in the public sector, why and how? The Netherlands Ministry of Finance 9th fee Public Sector Conference. Disponible en: <http://www.ecgi.org/codes/ documents/public_sector.pdf>. Acceso: 22/08/2012.

TONI, J. (2002): "Governança participativa e planejamento de longo prazo: o caso do governo do estado do Rio Grande do Sul, Brasil” en VII Congreso Internacional del CLAD sobre la Reforma del Estado y de la Administración Pública, Lisboa, Portugal, 8-11 Oct.

YIN, R. K (2011): Estudo de Caso: Planejamento e Método. 2. ed. São Paulo: Bookman.

YOUNG, I. M. (2006): “Representação política, identidade e minorias”, Lua Nova, São Paulo, n.67, pags.139190. 